\title{
Impact of big data on computer graphics
}

\author{
Pramila Joshi* \\ Birla Institute of Technology, Ext Centre, Noida, India
}

(C2017 ACCENTS

\begin{abstract}
Computer graphics is a field which has touched almost every arena in human life be it entertainment, latest GUIs, medical field, aeronautics, and what not. All the applications are computer graphics based. Big data as a trending feature today has also got great impact on computer graphics. In this paper we will discuss how the concepts of big data have impacted the various applications based on computer graphics. What are the latest tools and technologies to handle computer graphics applications with big data, and what are the opportunities and challenges being faced when computer graphic applications deal with huge volumes of data called big data. After having read and analysed various research papers on big data and its applications, it is observed that data visualization is very important in order to analyse the results produced from big data processing.
\end{abstract}

\section{Keywords}

Big data, Computer graphics, Data visualization, Hadoop, Geo spatial data.

\section{Introduction}

Computer graphics has touched many arenas in past twenty years ranging from studio arts to several mathematical fields. The disciplines which have the greatest impact of computer graphics in the form of special effects can be classified as advertising and entertainment industry, visual representation techniques and industrial architecture.

\section{Era of big data}

We are living in the era of big data. Big data holds no value until it is analyzed and more so visualized. In fact data visualizations has become very important in today's time and it is needed and used everywhere, be it an executive presentation using visual representation of data points as part of an executive presentation, to exhibit timely progress. Large scale data sets or big data sometimes is so complex that perceiving data and extracting meaningful information out of this huge ocean of data is difficult. Limited screen space available for displaying results also poses problems. There are methods which can be customized so that large scale data can be visualized and analyzed more quickly and efficiently [1].

\footnotetext{
*Author for correspondence
}

\section{Tools for visual representation}

One needs several tools in order to have impressive visual representation and designs and most of the tools are available free online, are open source and work in tandem with each other. The tools may not require any programming with a provision of drag and drop facility and are able to work with existing design. They use SVG, JavaScript, HTML5, Python or JSON. These tools work on platforms with a business intelligence backup to provide effective data analysis and impressive data visualization.

However, despite many recent advances, the scale, heterogeneity, and complexity of modern scientific datasets continue to pose major challenges for visualization designers. In many situations, there are just too few pixels to effectively visualize these datasets. Moreover, solving scientific problems today requires concentrated effort from interdisciplinary teams who deal with various data sources, including 3D, 2D, abstract, temporal, and multidimensional datasets. It's challenging to construct integrative visualizations that can simultaneously and effectively cater to such a variety of data sources [2].

\section{Sources of big data}

Sources of big data are mostly based on Computer graphics applications. Some of the sources are range scanning, imagery, sensor networks, scientific computing, light transport, databases and web. Most of them deal with one form of graphics or the other. 
Big data is no longer a new notion and can be seen as a continuous trend in modern technology. The term big data is applicable to all the information that can no longer be handled using traditional means. It is interesting to see this challenge in terms of big data being faced by the organizations today. They have access to mines of data and information but it is so much unstructured and unformatted and raw that they don't know how to extract value from this pool of data. As a matter of fact they don't even know if it is worth keeping or not. The result is that they don't have business insights they need for their growth. In today's scenario data generation has become a continuous process thanks to the widespread use of internet. Adding to the woes are automated systems, multiple sensors and what not. Social networking sites are also contributing to this big data problem. Big organizations active in Internet faced these problems and came up with extempore solutions to cope with the situation. In order to make these solutions available to public, open source software companies got hold of them gradually. And that was it. Big Data became a trend with easy and inexpensive solutions available commercially.

\section{Big data definition}

"Big Data" is a term encompassing the use of techniques to capture, process, analyse and visualize potentially large datasets in a reasonable timeframe not accessible to standard IT technologies. By extension, the platform, tools and software used for this purpose are collectively called "Big Data technologies". IBM has characterized Big Data in terms of Volume, Velocity and Variety;

\section{A. Volume}

The present age is the age of Data overload. People, Small scale companies and MNCs all store Data be it personal, business related or of miscellaneous nature as information is power. The volume of data thus has become massive moving from Terabytes to Petabytes and now from Petabytes to Zettabytes.

\section{B. Variety}

Since the data is being generated by and collected from a plethora of sources in different formats varying between structured and highly unstructured, the data processing itself is becoming increasingly complex.

\section{Velocity}

Velocity in the context of Big Data refers to the speed with which the data is generated and processed in support of decision making and deals with growing data production rates. It is becoming more and more real-time.

\section{What is computer graphics}

Computer Graphics is not a phenomenon which has a definition. In fact it is an area of computer application which deals with how to display images on computer screen. Computer Graphics actually started with the display of data on hardcopy plotters and CRT screens when the computer era began. Since then it has evolved to the extent of creation, storage and manipulation of models and images of objects. These models come from a diverse and expanding set of fields and include physical, mathematical, engineering, architectural and abstract structures, natural phenomenon and so on. CG today is largely interactive. The user controls the contents, structure and appearance of objects and their displayed images by using input devices such as keyboard mouse or touch panels on the screen. This is going to be an interesting concept as to how these two diverse fields can be integrated or can help each other.

\section{Big data in computer graphics}

To understand this let us first see the areas where computer graphics is used these days. We also side by side discuss the impact of big data on these fields. In the recent past wide ranging output screens are being used for visualizing enormous data (big data). They are usually made up of tiled LCD monitors to give a feel of smooth continuous surface. It has extremely high resolution to aid enormous diverse data to be envisaged efficiently. Scientists can make use of this technique to analyses their routing workflow. Tiled LCD walls are considered better as compared to CAVEs as far as the image quality is concerned. They offer higher image quality and better resolution up to thousand mega pixels [3].

\section{Applications of computer graphics}

Computer Graphics has numerous applications, some of which are highly affected by the advent of big data, listed below:

\section{A. Entertainment and Advertising}

Computer Graphics is widely used in entertainment industry to provide special effects and other support. It is used in form of multimedia which is highly interactive and does variety of things from audio, video and animation. It has become so vital in today's time that it can create or destroy any performance. Variety of tools is available which make it highly difficult if not impossible to differentiate between real and artificial objects/subjects used in pictures and films. 
Coming back to the connection of computer graphics with big data, this is a known fact that major source of entertainment today are smart phones and digital media being used in them. The graphics users and media creators must adapt to the new trends in big data analytics to discover the highly changing and highly dynamic customer insights which vary from time to time and region to region. Getting to understand customer behaviour is becoming highly difficult with their ever changing demands.

It is becoming a latest trend to post reviews and feedback on social media. Vast amount of structured and unstructured data in form of images and videos is getting accumulated from multiple sources and without taking help of big data tools and techniques it seems a next to impossible task to make sense of this huge ocean of data. Thanks to big data that helps predict the likes and dislikes behaviour and reaction of customers on latest programs and movies. It is not difficult to know why customers subscribe or unsubscribe. Entertainment channels and Media companies can optimize their returns and profits through scheduled media streaming.

Entertainment industry is evolving rapidly with their increased ability to analyse and act large volumes of customer generated big data. Their performance is based on their calibre to face the challenges in the fields of content distribution and promotion which is also a part of big data analytics. The more they are able to adapt to customer behaviour and preference, the more revenue they generate, the more successful they become. Another trend which big data has helped the entertainment industry understand is that consumers are becoming highly selective. They are doing away with subscription services and are more into buying or downloading the content and then viewing it in their own leisure time. It is becoming the need of the hour for media and entertainment companies to keep their data warehouses up to date and adopt new analytical data models which can scale up with incoming traffic and elapsed time. If we talk about big data in graphics, it is a collection of multiple two dimensional and three dimensional images being produced in abundance. How to view this data is a big challenge to the media industry today. A new technology cave2 has emerged which offers a new kind of visualization space. With these techniques it is possible to view multidimensional information at the same time, thus giving media industry more opportunities to grow [4].

\section{B. Scientific Visualization}

Though the name seems highly scientific and less glamorous than the above topic but it is another important field which has to deal with computer graphics and big data at the same time. This area is about computer generated models, and simulating and depicting their behaviour which is not possible otherwise because the objects in question are either too small or too big or too remote or too dangerous. For example chemical compound structures, global weather patterns, topography of distant planets, deep ocean pictures, hurricanes etc.

Scientific visualization means to be able to extract meaningful information from the huge ocean of data sets. One important aspect about visualizing big data is absence of interactivity. It has been observed that most interactive algorithms and techniques do not go well with big data therefore many times scientists have to remain contended with offline techniques only when working with big data sets.

One simple solution can be to first maximize data size and second maximize interactivity rather than choosing the reverse.

Big data is about magnitude. With time as it is scaling a manifold, the more complicated it is becoming to analyse and display of results. One needs better display screens to view these enormous datasets which are growing continuously. Massive displays with high resolution technology are something which has become latest research trend. New algorithms are being looked for designing interactive visual analytic interfaces [5].

\section{Cartography-drawing Maps}

Cartography is the process of drawing maps with the help of data gathered and evaluated. It is a complicated process and involves intelligent and high graphical techniques in order to produce the final map. The cartographer must possess the required skills, should have complete knowledge about drawing the map. The skills include collection, dissemination and analysis of geospatial data.

As far as geospatial big data is concerned, using it for cartography itself is a big challenge, keeping in mind the technical and computational problems associated with it. However cartography is inclined to make use of big data so as to improve its results. But leaving behind contemporary techniques and procedures would also not be a great idea. As far as geospatial big data is concerned, it offers lot of scope in the 
field of cartography. Cartography science is touching new realms and technical paradigms with the use of big data. New research agendas need to be developed which should focus on drawing maps based on geospatial big data. The agenda is challenging and needs to be paid ultimate attention [6].

\section{Weather Maps}

As we know that computer graphics is all about creating pictures and images using computers. Here we usually are talking about computer generated image data, which has been created by the assistance of some specialized graphics hardware and software. It's a vast area and comparatively a recent one in computer science.

At the same time big data is also an emerging trend and has been highly encouraged and adopted due to the latest advancement in technology. It defines new realms in the field of scientific analysis. With data storage prices slashed considerably, internet speed going up like never before and fast processors taking over the slow ones, have allowed scientists to use big data wherever possible. Weather forecasting is also making use of it as much as it can. Powerful supercomputers today can access big data efficiently and are able to do better and correct forecasts. Weather systems can also be modelled as so much of data is available with all the latest technology and procedures waiting to take shape.

As the trend goes, the large volumes of sensor datasets are processed using Hadoop with map reduce technology. This MapReduce framework can process huge volumes of data using parallel and distributed systems. Weather scientists are seeing great potential in using Hadoop technology in dealing with big data to help them do correct forecast. Goes without saying that correct weather forecast is highly beneficial for the humankind for they can preplan their tours and trips based on weather conditions of their destination. Weather forecasting problem include prediction of temperature, rain, fog, winds, clouds, storm etc. Weather sensors collect data every hour at many locations and gather a huge data. Weather forecasting is always a big challenge because it is hard to predict the state of the atmosphere for the upcoming future because climate dataset is unpredictable and frequently change according to global climate changes. The data used is from the national climatic data centre (NCDC), the format of dataset support a rich set of meteorological elements, which are good candidate for analysis with big data because it is semi-structured and record oriented [7].

\section{E. Satellite Imaging}

Another area where computer graphics is vastly used is satellite imaging, which is the backbone for information to meet departments. The satellites can be termed as eyes of the sky. Satellite images are the pictures of different parts of the earth by various angles taken by satellites. Understanding and analysing these pictures give met scientists a clear view about the events taking place on those areas of earth. Analysis of these images can prove very beneficial in the areas of cartography as we discussed earlier, military operations and weather predictions. But to be able to use these vast volumes of data available in form of images one needs specialized technical support, which is provided by big data technology. This field has a huge potential to provide timely statistical outputs and helps the met department to take more informed decisions. Also results in reduced surveys which cut down costs tremendously.

Various R\&D companies are working with GIS and turning the geospatial big data into smart data which can help in correct and informed decision making. Challenges related to big data technicalities are there but they are being overcome by new big data technologies.

For example how to handle, process and analyse image databases is a big challenge. Use of single computer also limits the possibilities. Hadoop comes as great help by its parallel and distributed computing environment. There is a need of a new parallel approach to work effectively on massed image data in-order to get the efficient results, which is possible by latest big data processing tools.

\section{F. Techniques to Analyse Video Data using Hadoop?}

Big data has come into being by the massive usage of smart phones and tablets all across the globe by the smart audience. Use of social networking sites has worked as fuel to generation of this massive data pool. People are uploading images and videos every second, even the sensors, cameras installed in public areas are producing image data every second. How to process this content is the biggest challenge today before the organizations. Let us look as to how the latest big data technology Hadoop offers a solution to this problem.

Hadoop converts the video into frames, put frames as stream of bytes in HDFS, storing images as HIPI 
image bundle, analysis of frames, analysis of image with HIPI API.

Since the data size is huge, clustering can be used as an important tool for data mining and knowledge discovery. Meaningful groups of data can be formed and analysed as a single entity [8].

\section{G.Engineering drawings-mechanical, Electrical,} civil, etc.-replacing the Blueprints of the Past

Engineering field cannot work without drawings and diagrams. Various types of drawings are needed to understand how calculations work and what result they produce. Showing the result in form of drawing and going back to calculations, programs and formulas if the result is not as expected, is the core of engineering science.

Drawing is important in all disciplines of engineering be it electrical, computer, civil or mechanical. Computer graphics has come to aid store these complex drawings inside the computers.

Now how has big data affected this application of computer graphics. Big data deals with huge volumes of data thus improving visibility of information. This improved visibility aids in design and manufacturing engineering. By analyzing trends one is able to predict inventory, manufacturing results, lifespan of the equipment used and cycles etc.

Big data mining results enable detect flaws in manufacturing design, suggest modifications based on past data and trends, thus saving design time and costs. Another benefit of big data mining in the field of engineering is to be able to respond rapidly to market trends, invent new products to match dynamic needs of clients, thus earning customer satisfaction.

\section{H.Architecture-construction plans, Exterior Sketches-replacing the Blueprints and Hand Drawings of the Past}

Architecture is another field which depends heavily on Computer Graphics. Big data has impacted this field also to a significant extent. This impact has not been acknowledged much surprisingly. However, having understood the importance of big data clients to the architectural firm are now stressing on more than just drawing sets as they have come to know about data rich BIM models that are used by management for project documentation. Clients want to know in advance how their design will look like.

\section{Challenges in big data visualization} Large high-resolution screens are becoming increasingly available and less expensive. This creates potential advantages for data visualization in that more dense data and fine details are viewable at once. Also, less navigation may be needed to see more data. However, little work has been done to determine the effectiveness of large high resolution displays, especially for basic low-level data visualization and navigation tasks. This paper describes an exploratory study on the effects of a large tiled display with a resolution of $3840 \times 3072$ as compared to two smaller displays (1560x2048 and $1280 x 1024)$. We conclude that, with finely detailed data, higher resolution displays that use physical navigation significantly outperform smaller displays that use pan and zoom navigation. Qualitatively, we also conclude that use of the larger display is less stressful and creates a better sense of confidence than the smaller displays [9].

Are computer graphics and big data connected? Yes through data visualization. Data visualization is the branch of computer graphics and to display the results after extracting patterns out of big data requires data visualization. If you present before the analysts results in the form of texts and data in several rows they won't be able to make any sense out of that. Pictorial analysis of results is easier to understand. But using graphs and templates on sample data which is less in volume will defeat the purpose of big data.

Now the real challenge comes in the form of the task as to how to represent huge volumes of big data so that they are visualized in the most optimum way. There is a need to understand the tools and techniques which help in this task.

\section{Conclusion and future work}

Data is becoming extremely important as more and more scientific areas and fields are evolving. Data analytics with visualization facility is becoming part of parcel of every business as more and more data can be analyzed with new big data analysis tools. Information presented in visual form as compared to textual form holds more value. It is better understandable. However when visualization techniques are combined with big data it has to meet many challenges, as both the fields themselves come across many challenges. Organizations need to be better equipped to handle these challenges to taste success in the strategy to combine big data with graphics. Using big data intelligence for growth of 
business is a wise decision and to be able to view the basis of decision making will be entirely new experience. If data is very large one can use various clustering techniques to analyses and manage the data efficiently.

\section{Acknowledgment}

None.

\section{Conflicts of interest}

The author has no conflicts of interest to declare.

\section{References}

[1] Choo J, Park H. Customizing computational methods for visual analytics with big data. IEEE Computer Graphics and Applications. 2013; 33(4):22-8.

[2] Reda K, Febretti A, Knoll A, Aurisano J, Leigh J, Johnson A, et al. Visualizing large, heterogeneous data in hybrid-reality environments. IEEE Computer Graphics and Applications. 2013; 33(4):38-48.

[3] Alper B, Hollerer T, Kuchera-Morin J, Forbes A. Stereoscopic highlighting: 2D graph visualization on stereo displays. IEEE Transactions on Visualization and Computer Graphics. 2011; 17(12):2325-33.
[4] Febretti A, Nishimoto A, Thigpen T, Talandis J, Long L, Pirtle JD, et al. CAVE2: a hybrid reality environment for immersive simulation and information analysis. In Is\&t/spie electronic imaging 2013 (p. 864903). International Society for Optics and Photonics.

[5] Reda K, Johnson A, Mateevitsi V, Offord C, Leigh J. Scalable visual queries for data exploration on large, high-resolution 3D displays. In high performance computing, networking, storage and analysis 2012 (pp. 196-205). IEEE.

[6] Robinson AC, Demšar U, Moore AB, Buckley A, Jiang B, Field $\mathrm{K}$, et al. Geospatial big data and cartography: research challenges and opportunities for making maps that matter. International Journal of Cartography. 2017; 11:1-29.

[7] Doreswami ,Gad I. Big data techniques: hadoop and map reduce for weather forecasting. International Journal of Latest Trends in Engineering and Technology, special issue SACAIM. 2016.

[8] Sreedhar C, Kasiviswanath N, Reddy PC. Clustering large datasets using k-means modified inter and intra clustering (KM-I2C) in Hadoop. Journal of Big Data. 2017; 4(27): 1-19.

[9] Ball R, North C. Effects of tiled high-resolution display on basic visualization and navigation tasks. In CHI'05 extended abstracts on human factors in computing systems 2005 (pp. 1196-9). ACM. 